\title{
Caractéristiques morpho-pédologiques et potentialités agricoles de sols développés sur roches dolomitiques dans le bassin de Taoudéni au Burkina Faso
}

\author{
Fidèle KABORE ${ }^{1 *}$, Edmond HIEN ${ }^{1,2}$, Tockville B. MARE ${ }^{1}$, Korodjouma OUATTARA ${ }^{3}$ et \\ Prosper N. ZOMBRE ${ }^{1}$
}

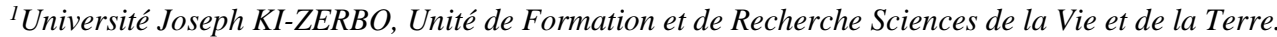 \\ Laboratoire Sols, Matériaux et Environnement (LSME), 03 BP 7021 Ouagadougou 03, Burkina Faso. \\ ${ }^{2}$ Institut de Recherche pour le Développement (IRD), UMR 210 Eco\&Sols, 2 Place Viala, 34060 Montpellier \\ Cedex 2, France. \\ ${ }^{3}$ Institut de l'Environnement et de Recherches Agricoles (INERA), 04 BP 8645 Ouagadougou 04, \\ Burkina Faso. \\ *Auteur correspondant ; E-mail : fidele_kabore@hotmail.com
}

\section{RESUME}

Dans l'Ouest du Burkina Faso ont été identifiés des sols développés sur des dolomies. L'absence d'étude pédologique antérieure dans la région, traitant de l'implication géochimique des roches dolomitiques sur les propriétés de ces sols, a conduit à la réalisation d'une étude morpho-pédologique. Celle-ci avait pour objectif d'évaluer l'impact des carbonates sur les caractéristiques morphologiques, physiques, chimiques et hydriques des sols en vue d'en dégager les potentialités. Cette prospection menée à partir de trois transects, partant chacun des trois principales collines dolomitiques de la région (Diounkan, Souroukoudinga et Samendéni) indique l'existence d'argiles de type 2/1. Ces argiles interviennent positivement dans la structuration des sols, la rétention de l'eau et des nutriments grâce à leur CEC élevée comprise entre 11 et $20 \mathrm{cmol}^{(+)} \cdot \mathrm{kg}^{-1}$. On note, par ailleurs, un déséquilibre cationique entre $\mathrm{Ca}^{2+}$ et $\mathrm{Mg}^{2+}$, car le ratio $\mathrm{Ca}^{2+} / \mathrm{Mg}^{2+}>1$ dans l'horizon organo-minéral, devient $<1$ en profondeur, traduisant ainsi un excès de $\mathrm{Mg}^{2+}$ à ce niveau. Les rapports $\mathrm{Mg}^{2+} / \mathrm{K}^{+}$et $\mathrm{Ca}^{2+} / \mathrm{K}^{+}$parfois $>50$ et le ratio $\mathrm{K}^{+} / \mathrm{CEC}<0,05$, indiquent une carence en $\mathrm{K}^{+}$. Par conséquent, le potassium pourrait constituer, avec le phosphore $\left(<5 \mathrm{mg} \cdot \mathrm{kg}^{-1}\right)$, les principaux facteurs limitant la productivité des sols. L'utilisation durable de ces sols exige un choix judicieux des types de cultures et un mode de fertilisation organo-minérale pour compenser les carences.

(C) 2020 International Formulae Group. All rights reserved.

Mots clés: Argiles, cations, antagonisme potassium-magnésium, fertilité du sol, roches dolomitiques.

\section{Morpho-pedological characteristics and agricultural potentialities of soils developed on dolomitic rocks in the Taoudeni basin in Burkina Faso}

\begin{abstract}
In western of Burkina Faso, soils developed on dolostones have been identified. Lack of previous soil study in the region, dealing with the geochemical implication of dolomitic rocks on the properties of these soils, led to the realization of a morpho-pedological study. The purpose of this study was to assess the impact of carbonates on the morphological, physical, chemical and water characteristics of the soils in order to identify
\end{abstract}


their potential. This prospecting carried out starting from three transects, starting each from the three main dolomitic hills of the region (Diounkan, Souroukoudinga and Samendeni) indicates the existence of type 2/1 clays. These clays play a positive role in the structuring of soils, the retention of water and nutrients thanks to

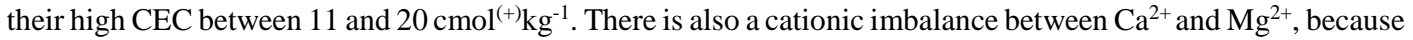
$\mathrm{Ca}^{2+} / \mathrm{Mg}^{2+}>1$ ratio in the organo-mineral horizon becomes $<1$ from the median horizon, thus reflecting an excess of $\mathrm{Mg}^{2+}$ at this level. The $\mathrm{Mg}^{2+} / \mathrm{K}^{+}$and $\mathrm{Ca}^{2+} / \mathrm{K}^{+}$ratios sometimes $>50$ and the $\mathrm{K}^{+} / \mathrm{CEC}$ ratio $<0.05$ indicate a deficiency of $\mathrm{K}^{+}$. Consequently, potassium could constitute with phosphorus $\left(<5 \mathrm{mg} . \mathrm{kg}^{-1}\right)$ the main factors limiting the productivity of these soils. The sustainable use of these soils requires a judicious choice of crop types and an organo-mineral fertilization method to compensate for the deficiencies.

(C) 2020 International Formulae Group. All rights reserved.

Keywords: Clay, cations, potassium-magnesium antagonism, soil fertility, dolostones.

\section{INTRODUCTION}

La dissolution de la calcite et/ou de la dolomite des roches carbonatées aboutit à l'accumulation de produits silicatés essentiellement constitués de feldspaths, de quartz et de minéraux argileux (Prudente et al., 2002). Ces silicates et certains éléments en particulier ( $\mathrm{Fe}$ et $\mathrm{Al}$ ), de teneur initialement faible à négligeable dans la roche, prennent de l'importance et orientent suivant le climat les caractéristiques du futur sol (Baize et al., 2016). La minéralogie et la granulométrie des résidus de décarbonatation déterminent la plupart des propriétés du sol. Lorsque les silicates libérés sont dominés par le quartz, les sols acquièrent une texture sableuse. Par contre, les sols sont plus argileux et plus lourds lorsque les minéraux argileux sont dominants (Baize et al., 2016). Quant aux deux principaux éléments majeurs des carbonates $\left(\mathrm{Ca}^{2+}\right.$ et $\mathrm{Mg}^{2+}$ ), ils sont entraînés soit en profondeur par les eaux de percolation, soit en bas de versant par le ruissellement (Koulibaly et al., 2014). La mobilité de ces cations et leur probable influence sur les propriétés des sols environnant les dolomies de la partie Ouest du Burkina Faso ont été soulignées par Bunasols (2002). L'argile provenant de la décarbonatation de ces dolomies génère des sols de teinte foncée en bas de versant des collines dolomitiques. Cependant, nous n'avons pas connaissance de travaux donnant une idée quantitative du degré d'influence des deux cations $\left(\mathrm{Ca}^{2+}\right.$ et $\left.\mathrm{Mg}^{2+}\right)$ libérés sur les paramètres morphologiques, physiques et chimiques des sols environnant les collines dolomitiques. Alors que des auteurs comme (Hannan, 2011; Akassimadou et Yao-Kouamé, 2014) ayant travaillé sur le $\mathrm{Ca}^{2+}$ et $\mathrm{Mg}^{2+}$ des sols ont constaté qu'une très grande quantité de l'un des deux cations, sur le complexe adsorbant du sol pouvait limiter l'adsorption de l'autre par les racines. D'ailleurs, des auteurs tels (Hamza, 2008 ; Ahukaemere et al., 2018) mentionnent que dans les sols riches en carbonates, l'abondance des ions $\mathrm{Ca}^{2+}$ et $\mathrm{Mg}^{2+}$ peut limiter la teneur des ions $\mathrm{K}^{+}$sur le complexe adsorbant qui devient dès lors, un élément limitant la productivité des sols. La présente étude a pour objectif général de contribuer à la connaissance des sols développés sur matériau carbonaté.

\section{MATERIEL ET METHODES}

\section{Description de la zone d'étude}

La présence d'affleurements de roches carbonatées a été le principal critère pour le choix de la zone d'étude. L'existence de ce type de roches dans cette région sédimentaire du Burkina Faso avait été déjà signalée par Jonquet (1963) et Hugot \& Carbonnel (1986). L'étude a été réalisée dans la région Ouest du Burkina Faso, aux alentours de BoboDioulasso. Les sites retenus sont Samendéni 
$\left(11^{\circ} 29 \mathrm{~N} ; 4^{\circ} 33 \mathrm{~W}\right)$, Souroukoudinga $\left(11^{\circ} 13 \mathrm{~N}\right.$; $\left.4^{\circ} 32 \mathrm{~W}\right)$ et Diounkan à Tiara $\left(11^{\circ} 00 \mathrm{~N}\right.$; $4^{\circ} 39 \mathrm{~W}$ ), présentant tous, des collines dolomitiques (Figure 1). Le climat est de type soudanien, marqué par une longue saison sèche et une courte saison de pluie. La pluviométrie moyenne annuelle est supérieure à $900 \mathrm{~mm}$. La température moyenne est de $35{ }^{\circ} \mathrm{C}$ pour les maxima et $19{ }^{\circ} \mathrm{C}$ pour les minimas. La végétation comprend une forêt galerie de Mitragyna inermis (Willd.) Kuntze, Ficus platyphylla Delile, Berlinia grandiflora (Vahl) Hutch. \& Dalziel, Terminalia macroptera Guill. \& Perr., etc. et des espèces forestières de vergers composées de Mangifera indica L. [cult.], Psidium guajava L. [cult.], Anacardium occidentale L. [cult.], Carica papaya L. [cult.]; une savane arbustive, dominée par Lannea microcarpa (Engl. \& K. Krause), Piliostigma reticulatum (DC.) Hochst., Guiera senegalensis J.F.Gmel., Diospyros mespiliformis Hochst. ex A.DC., Combretum micranthum G.Don, Acacia seyal Delile, Parkia biglobosa (Jacq.) R.Br. ex G.Don, Khaya senegalensis (Desr.) A.Juss., Tamarindus indica L., etc. et une savane herbeuse formée de: Andropogon gayanus Kunth, Andropogon chinensis (Nees) Merr., Cymbopogon schoenanthus (L.) Spreng., Chrysopogon nigritanus (Benth.) Veldkamp, etc.

La géomorphologie de la région est formée de plateaux latéritiques cuirassés, de larges vallées (plaines et dépressions périphériques) et quelques buttes et collines. Le réseau hydrographique est formé par le fleuve Mouhoun et ses affluents. Les formations géologiques sont les grès de base, les dolérites et les carbonates qui affleurent de façon discontinue dans deux formations stratigraphiques de la région (Guéna-Souroukoudinga et SamendéniKiébani).

\section{Echantillonnage des roches carbonatées}

Six échantillons de roche ont été prélevés des affleurements de dolomie (Figure 1). Ces matériaux prélevés en surface pourraient expliquer certaines caractéristiques des sols issus de leur altération.

\section{Ouverture des transects}

Trois transects (Figure 1) ont été suivis pour réaliser des fosses pédologiques sur les trois principales collines carbonatées de la région (Jonquet, 1963). Le choix des transects et de la position des fosses a tenu compte des critères géologique, géomorphologique et pédologique. En effet, le transect part toujours du sommet de la colline dolomitique et se limite dans la vallée (plaine ou dépression périphérique) qui correspond aux points de basses altitudes. La direction du transect est choisie de manière à traverser les deux grandes classes de sols (les lithosols et les sols sesquioxydes de fer et de manganèse) répertoriées par Bunasols (2002) sur et autour de chacune des collines dolomitiques. Au moins une observation pédologique est effectuée à chaque $10 \mathrm{~m}$ de dénivelé.

\section{Description des fosses et échantillonnage}

Les différents sols ont été décrits en saison sèche à l'aide d'une fiche de description pédologique qui permet de noter les caractéristiques morphologiques importantes (détermination de l'épaisseur des horizons à l'aide d'un mètre ruban, de la couleur à l'aide du code Munsell, appréciation à la vue et au toucher de la texture, de la structure du sol, du taux et de la nature des éléments grossiers, de la consistance, de la transition entre horizons, etc.).

Dans chaque fosse pédologique, les horizons ont été regroupés en trois types: l'horizon de surface ou horizon organo-minéral "A"; l'horizon médian qui peut être un horizon structuré "(B)" ou un horizon 
d'accumulation "B" et enfin l'horizon "C" qui correspond à l'horizon d'altération situé audessus de la dolomie pour les sols peu profonds. En ce qui concerne les sols profonds, "C" désigne l'horizon d'apparition des cailloux dolomitiques provenant de la dolomie sousjacente. L'acide chlorhydrique $(\mathrm{HCl})$ dilué à $10 \%$ a été utilisé pour tester la présence de calcaire actif dans les horizons de sol. Dans chaque horizon, trois faces ont été échantillonnées puis mélangés de sorte à obtenir un échantillon composite. Les sols décrits ont été classés selon la nomenclature Base de Référence Mondiale des sols (IUSS Working Group WRB, 2015).

\section{Travaux de laboratoire}

La géochimie totale de la dolomie a été réalisée par la fluorescence des rayons $\mathrm{X}$ (XRF) sur des échantillons de roches broyés à 75 microns (Ahamad et al., 2009).

Les échantillons composites de sols ont été tamisés respectivement à $4 \mathrm{~mm}, 2 \mathrm{~mm}$ et 0,5 $\mathrm{mm}$. Le lot de $2 \mathrm{~mm}$ a servi pour l'analyse de la granulométrie à 5 fractions selon la méthode de la pipette Robinson. Le refus minéral (particules minérales de diamètre $>2 \mathrm{~mm}$ ) a été pesé et le taux de refus a été déterminé par la formule:

Refus minéral

$=\left(\frac{\text { poids de refus minéral }}{\text { poids d'échantillon composite }}\right) * 100$

Poids refus minéral et échantillon composite (en g); Refus minéral (en \%)

Les taux de cailloux dolomitiques et de graviers ferrugineux ont été estimés en considérant la quantité de chacun dans le refus minéral.

La fraction de $4 \mathrm{~mm}$ a été utilisée dans la détermination de la réserve utile en eau "RU" des différents horizons des sols à partir des valeurs du $\mathrm{pF} 2,5$ et $\mathrm{pF}$ 4,2 déterminées par la méthode marmite de RICHARD. La formule suivante a été utilisée:

$R U=(p F 2,5-p F 4,2) * d a * z$

$\boldsymbol{R} \boldsymbol{U}$ : réserve en eau utile (en $\mathrm{mm}$ ); $\boldsymbol{d a}$ : densité apparente du sol, en moyenne de 1,7 pour des sols du Burkina Faso (Bunasols, 2002); $z$ : épaisseur de l'horizon (en $\mathrm{dm}$ )

La fraction de $2 \mathrm{~mm}$ a aussi servi pour déterminer: le taux de matière organique "MO" selon la méthode Walkley et Black (1934); l'azote total "N" par la méthode Kjeldahl ; le $\mathrm{pH}$ eau par la méthode électrométrique à l'aide d'un $\mathrm{pH}$-mètre à électrode en verre dans une suspension sol/eau (rapport 2/5); et enfin le phosphore assimilable ( $\mathrm{P}$ assimilable) par la méthode Bray I. La fraction $0,5 \mathrm{~mm}$ a servi pour le dosage des bases échangeables $\left(\mathrm{Ca}^{2+}\right.$ et $\mathrm{Mg}^{2+}$ par spectrométrie en absorption atomique, et en émission de flamme pour $\mathrm{Na}^{+}$ et $\mathrm{K}^{+}$) et de la capacité d'échange cationique "CEC" après extraction à l'aide d'une solution d'argent thiourée à $0,01 \mathrm{M}$.

\section{Réalisation des profils topographiques}

Les cartes ont été élaborées grâce au logiciel Arcgis 10.2. Les profils topographiques des trois transects ont été extraits à partir du Modèle Numérique de Terrain (MNT) de la zone d'étude à l'aide du logiciel Global Mapper 16.

\section{Analyses statistiques}

Les Tableaux ont été élaborés à l'aide du tableur Microsoft Excel 2010. Les analyses statistiques (moyennes et test de corrélation de Spearman au seuil de probabilité $p=0,05$ ) des données collectées ont été réalisées à partir du logiciel Stata. 


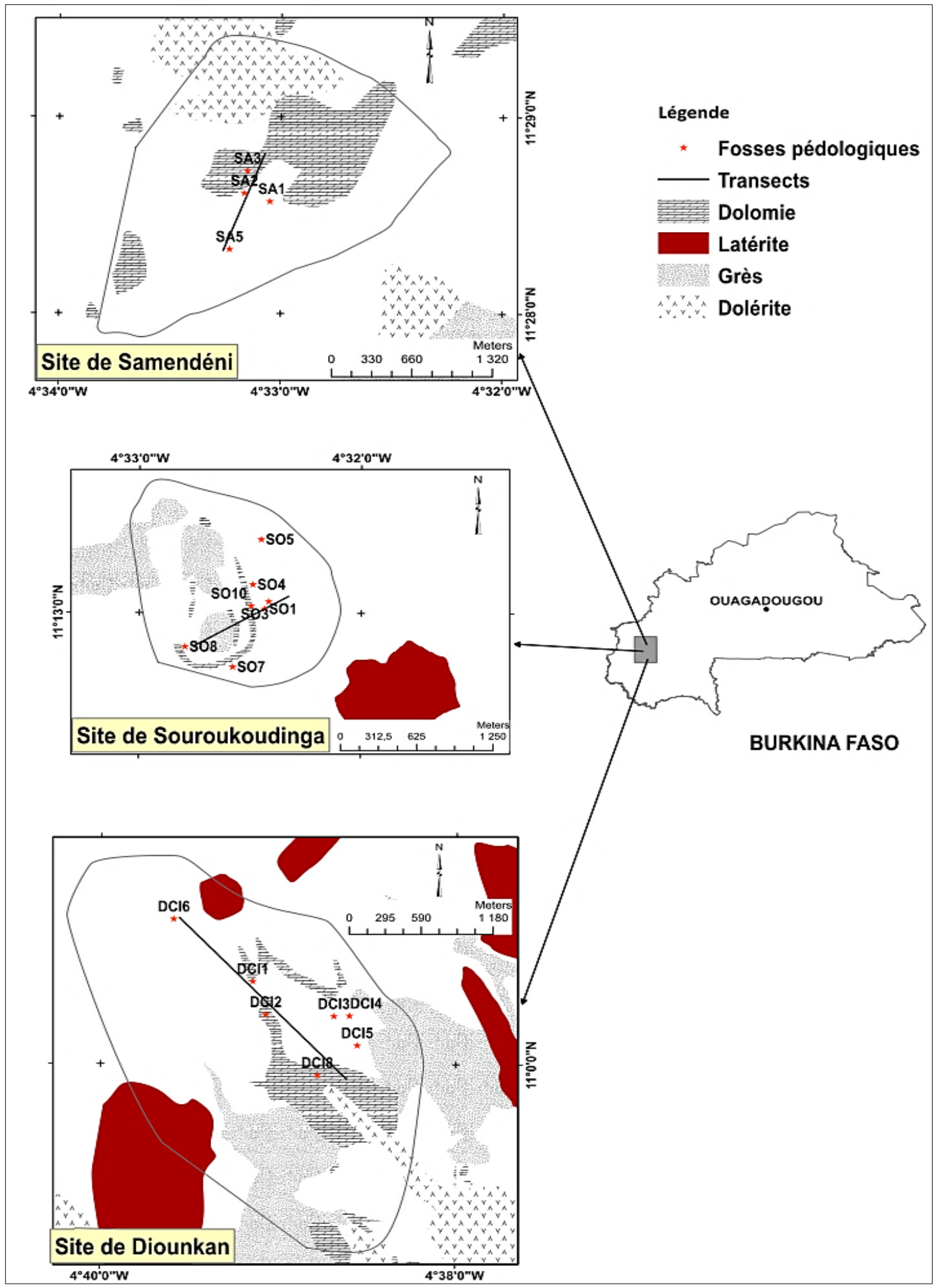

Figure 1 : Situation géographique, géologie et position des transects dans la zone d'étude. 


\section{RESULTATS}

\section{Caractéristiques de la roche carbonatée}

Les données chimiques (Tableau 1) montrent que les oxydes de calcium et de magnésium $(\mathrm{CaO}+\mathrm{MgO})$ et la silice $\left(\mathrm{SiO}_{2}\right)$ sont les principaux constituants de la roche. En revanche, les oxydes de fer $\left(\mathrm{Fe}_{2} \mathrm{O}_{3}\right)$, d'aluminium $\left(\mathrm{Al}_{2} \mathrm{O}_{3}\right)$, de potassium $\mathrm{K}_{2} \mathrm{O}$ et de phosphore $\left(\mathrm{P}_{2} \mathrm{O}_{5}\right)$ de la roche sont faibles. Quant aux oxydes de titane $\left(\mathrm{TiO}_{2}\right)$ et de manganèse $\left(\mathrm{MnO}_{2}\right)$, leurs teneurs sont encore plus faibles dans la roche. La valeur du $\mathrm{pH}$ eau révèle que la roche est moyennement alcaline.

\section{Caractéristiques morphologiques et physiques des sols}

Suivant les trois transects (Figures 2, 3 et 4), cinq types de sols ont été identifiés au voisinage des collines dolomitiques.

Dans le site Diounkan, les Leptisols lithiques (sols superficiels) occupent le sommet de la colline dolomitique et une portion du versant. Dans la plaine à pente relativement plus faible se développent des Regosols formés essentiellement de colluvions de la dolomie située en amont. Les Regosols sont progressivement remplacés en aval par des Cambisols vertiques. Ces Cambisols s'étendent jusqu'au bas-fond où ils forment des Cambisols vertiques endogleyiques (saturés d'eau en saison pluvieuse). Les Cambisols vertiques situés dans les niveaux les plus bas du site (Figures 2) sont les plus profonds et les plus riches en éléments fins (argile et limon).

Dans le site de Souroukoudinga, le sommet et une portion du versant de la colline sont tenus par les Leptisols lithiques, l'autre portion $\mathrm{du}$ versant allant vers le bas, caractérisée par une pente bien marquée est occupée par les Cambisols vertiques chromiques (Figures 3). Les Cambisols vertiques de teinte plus foncée se développent dans la dépression périphérique. Dans ce site également, les Cambisols vertiques deviennent progressivement des Cambisols vertiques endogleyiques dans le bas-fond. Dans la dépression, les sols sont plus profonds.

A Samendéni, le sommet de la colline et une partie du versant sont occupés par les
Leptisols lithiques. Les Cambisols vertiques chromiques qui succèdent aux Leptisols en aval sont bien drainés au regard de la pente relativement bien prononcée. La pente reste bien marquée jusqu'au bas-fond, occupé par des Cambisols vertiques endogleyiques. Dans ce milieu dominé par les Cambisols, se développent localement des Lixisols ferriques sur les «éboulis latéritiques » (Figures 4).

NB: Les "éboulis latéritiques" abordés dans cette étude sont des recouvrements récents formés essentiellement de colluvions (graviers, cailloux et blocs ferrugineux) issus $d u$ démantèlement de la cuirasse des plateaux environnants. Ces matériaux tapissent par endroits les versants des collines dolomitiques.

\section{Regosols colluviques}

Du point de vue morphologique, les Regosols colluviques identifiés sur le versant de la colline de Diounkan (DCI8) sont surtout caractérisés par les nombreuses colluvions dolomitiques provenant du haut de la colline (Tableau 2).

\section{Cambisols vertiques chromiques}

Ces Cambisols décrits en haut de versant des collines de Souroukoudinga (SO10) et de Samendéni (SA3 et SA2) montrent une coloration rouge même à l'état humide (Tableau 3).

\section{Cambisols vertiques}

Les Cambisols vertiques répertoriés en bas de versant des collines de Diounkan (DCI1) et de Souroukoudinga (SO1) ont des horizons faiblement différenciés. Par ailleurs, le passage d'un horizon à l'autre se fait progressivement (Tableau 4).

\section{Cambisols vertiques endogleyiques}

Ces Cambisols identifiés dans les basfonds en aval des collines de Diounkan (DCI6), de Souroukoudinga (S01) et de Samendéni (SA5) sont caractérisés par une l'hydromorphie temporaire et la présence de nombreuses taches d'oxydo-réduction dès l'horizon médian (Tableau 5). 


\section{Lixisols ferriques sur éboulis latéritique}

Le taux de graviers ferrugineux est très élevé depuis la surface jusqu'en profondeur dans les Lixisols (Tableau 6) décrits en bas de versant de la colline de Samendéni (SA1). Les quelques cailloux de dolomie de colluvionnement observables à la surface du sol, disparaissent dans l'horizon B. Ceux-ci ne réapparaissent qu'en profondeur à la lisière de la roche carbonatée sous-jacente.

\section{Paramètres hydriques des sols}

Les données hydriques présentées dans le Tableau 7 représentent une moyenne des caractéristiques hydriques dans chaque horizon de la classe de sols considérée.

Les humidités caractéristiques $\mathrm{pF} 2,5$; $\mathrm{pF} 4,2$ et $\mathrm{RU}$ sont en général plus élevées dans les Cambisols vertiques, très profonds et riches en éléments fins. Dans l'horizon B de ces Cambisols vertiques, la RU moyenne calculée dépasse $180 \mathrm{~mm}$ sur une épaisseur (z) moyenne d'environ $120 \mathrm{~cm}$. Les sols de versants de colline (Regosols colluviques et Cambisols vertiques chromiques) peu profonds et d'horizons peu épais montrent les plus faibles RU dans leur horizon B. Les valeurs inscrites dans le Tableau 8 sont des coefficients de corrélation de Spearman au seuil de probabilité $(p=0,05)$. Ces corrélations entre les paramètres hydriques, granulométriques et chimiques des sols ont été faites avec $n=45$ échantillons.

Les humidités caractéristiques à $\mathrm{pF} 2,5$ et $\mathrm{pF} 4,2$ sont liées positivement et significativement aux taux d'argile, de limon fin, à l'ensemble argile + limon fin et à la CEC. La RU est aussi liée positivement à l'argile et à l'assemble argile + limon fin. La matière organique est corrélée négativement au taux d'argile. Les sables fins et sables grossiers sont négativement corrélés aux humidités caractéristiques à $\mathrm{pF} 2,5$ et $\mathrm{pF} 4,2$.

\section{Caractéristiques chimiques des sols}

Les paramètres chimiques répertoriés dans le Tableau 9 sont obtenus à partir de la moyenne des paramètres chimiques dans chaque horizon de la classe de sols considérée.

Les taux de matière organique (MO) des sols sont moyens à faibles en surface. L'azote total $(\mathrm{N})$ reste moyen dans cet horizon organominéral. Les teneurs de ces deux paramètres chimiques diminuent en profondeur. Les sols issus de la dolomie sont plus pourvus en bases échangeables (Tableau 9) et la CEC des sols est moyenne à élevée sauf dans les Lixisols.

L'ordre de prédominance des bases à partir de l'horizon B est particulier: $\mathrm{Mg}^{2+}>$ $\mathrm{Ca}^{2+}>\mathrm{K}^{+}>\mathrm{Na}^{+}$; en général, $\mathrm{Ca}^{2+}>\mathrm{Mg}^{2+}$. Les ratios $\mathrm{Ca}^{2+} / \mathrm{Mg}^{2+}, \mathrm{Mg}^{2+} / \mathrm{K}^{+}$et $\mathrm{K}^{+} / \mathrm{CEC}$ indiquent que les sols sont enrichis en magnésium (Tableau 10).

Les sols sont appauvris en potassium disponible mais aussi en phosphore assimilable au regard des teneurs en cet élément dans l'horizon organo-minéral (Figure 5).

Les teneurs en $\mathrm{P}$ assimilable de horizon organo-minéral des différents types de sols sont très faible $\left(<5 \mathrm{mg} \cdot \mathrm{kg}^{-1}\right)$. Les sols étudiés sont tous carencés en phosphore assimilable.

Tableau 1: Caractéristiques chimiques de la roche carbonatée.

\begin{tabular}{|c|c|c|c|c|c|c|c|c|c|}
\hline $\mathrm{CaO}$ & MgO & $\mathrm{SiO}_{2}$ & $\mathrm{Fe}_{2} \mathrm{O}_{3}$ & $\mathbf{A l}_{2} \mathbf{O}_{3}$ & $\mathbf{K}_{2} \mathrm{O}$ & $\mathbf{P}_{2} \mathbf{O}_{5}$ & $\mathrm{TiO}_{2}$ & $\mathrm{MnO}_{2}$ & pH eau \\
\hline \multicolumn{10}{|c|}{$(\%)$} \\
\hline $29,13 \pm$ & 16,81 & 4,30 & 1,61 & $1,44 \quad \pm$ & 1,07 & 0,82 & 0,08 & $0,04 \pm$ & 8,0 \\
\hline 1,83 & $\pm 2,74$ & $\pm 2,49$ & $\pm 0,78$ & 0,45 & $\pm 0,83$ & $\pm 0,07$ & $\pm 0,04$ & 0,02 & $\pm 0,4$ \\
\hline
\end{tabular}




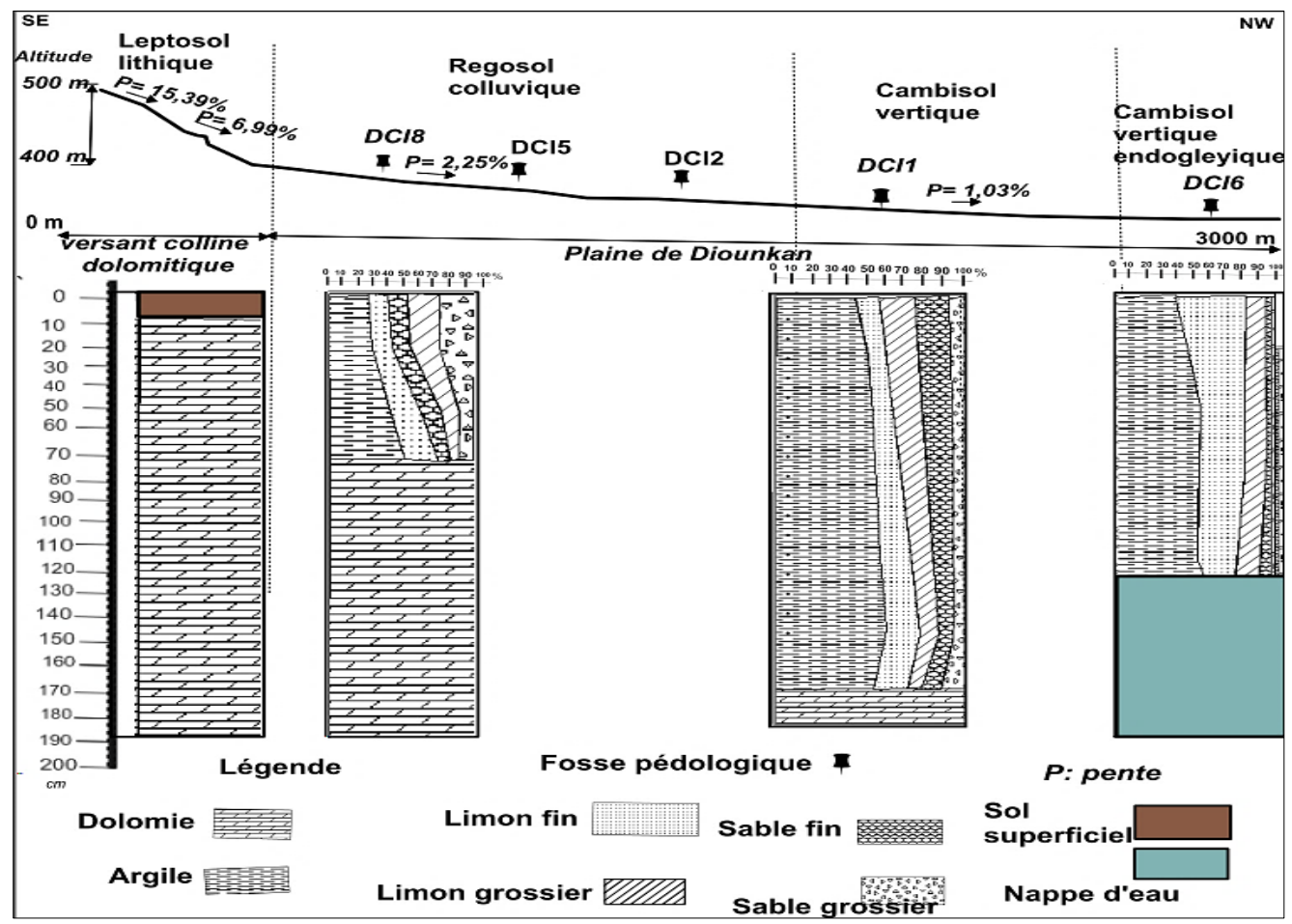

Figure 2: Transect de Diounkan et granulométrie des sols suivant la topographie.

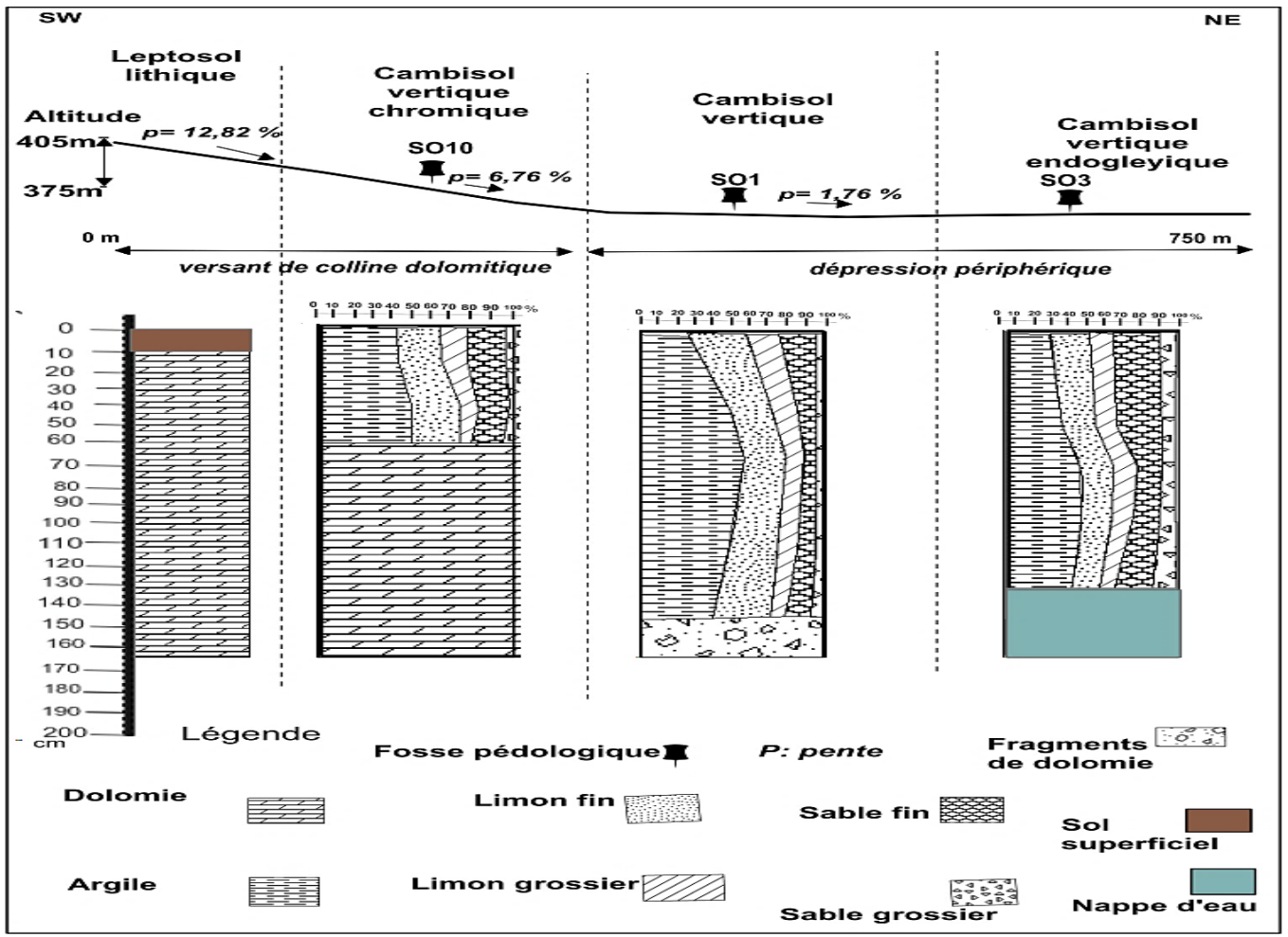

Figure 3: Transect de Souroukoudinga et granulométrie des sols suivant la topographie. 


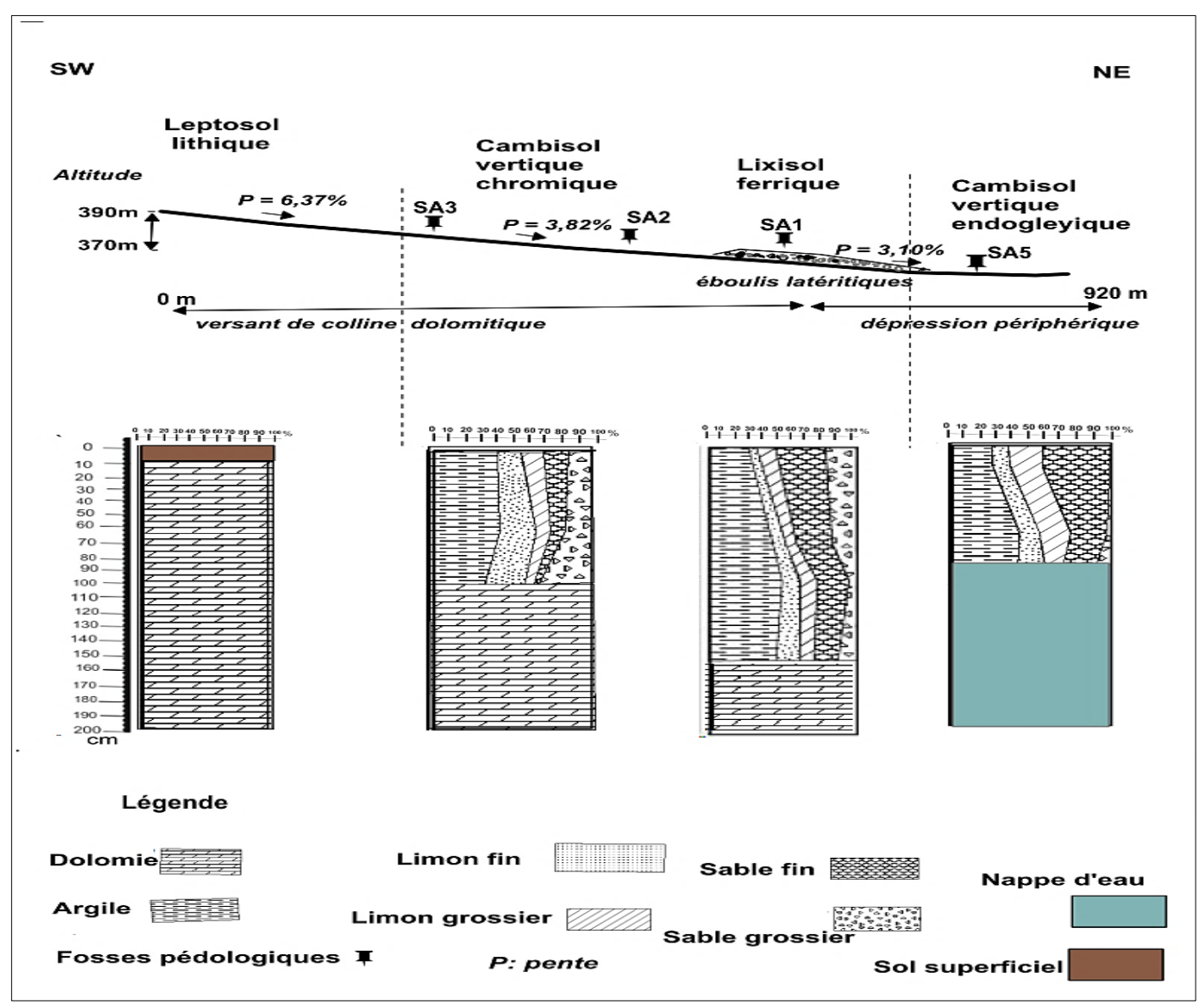

Figure 4: Transect de Samendéni et granulométrie des sols suivant la topographie.

Tableau 2: Description morphologique d'un profil type de Regosols colluviques à Diounkan.

\begin{tabular}{llll}
\hline Horizon & A & (B) & C \\
\hline Epaisseur (cm) & 0 à 20 & 20 à 60 & 60 à100 \\
\hline Couleur & 2,5 Y 3/2 & 10 YR 7/8 & 7,5 YR 5/6 \\
\cline { 2 - 4 } & brun grisâtre très sombre & jaune & bun foncé \\
\hline Taches & néant & néant & néant \\
\hline Texture & limono-argileuse & argileuse & argileux \\
\hline Structure & massive & massive & massive \\
\hline Porosité & nombreux pores tubulaires et & peu de pores tubulaires et & très peu de pores \\
& peu de fines fentes de retrait & fines fentes de retrait & \\
\hline Refus (\%) & 10 & 50 & 48 \\
\hline Grav. Ferrug. (\%) & 2 & 2 & néant \\
\hline Caill. dolom. (\%) & 98 & 98 & 100 \\
\hline Consistance & friable & peu dure & peu dure \\
\hline Test HCl & pas effervescence & pas effervescence & pas effervescence \\
\hline Transition & discontinue & discontinue & - \\
\hline $\begin{array}{l}\text { Texture (triangle USDA) } \\
\text { dolom.: Cailloux dolomitiques ; } \text { Test HCl: Réaction de la terre fine avec le HCl (10\%) à froid. }\end{array}$ &
\end{tabular}


Tableau 3: Description morphologique d'un profil type de Cambisols vertiques chromiques à Souroukoudinga.

\begin{tabular}{llll}
\hline Horizon & A & (B) & C \\
\hline Epaisseur (cm) & 0 à 20 & 20 à 50 & 50 à 70 \\
\hline Couleur & 5 YR 3/2 & 5 YR 6/4 & 5 YR 6/4 \\
\cline { 2 - 4 } & brun rougeâtre sombre & brun rougeâtre claire & brun rougeâtre claire \\
\hline Taches & néant & néant & néant \\
\hline Texture & limono-argileuse & argileuse & argileuse \\
\hline Structure & massive & polyédrique à & polyédrique à \\
& & assemblage compact & assemblage compact \\
\hline Porosité & nombreux pores tubulaires et & peu de pores & peu de pores \\
& fines fentes de retrait & tubulaires & tubulaires \\
\hline Refus (\%) & 13 & 40 & 54 \\
\hline Grav. Ferrug. (\%) & 40 & néant & néant \\
\hline Caill. dolom. (\%) & 60 & 100 & 100 \\
\hline Consistance & peu dure ; & dure & très dure \\
\hline Test HCl & pas effervescence & pas effervescence & pas effervescence \\
\hline Transition & progressive & progressive & - \\
\hline
\end{tabular}

Texture (triangle USDA) ; Couleur (Code Munsell) ; Refus : Refus minéral ; Grav. Ferrug.: Graviers ferrugineuses ; Caill. dolom.: Cailloux dolomitiques ; Test HCl: Réaction de la terre fine avec le $\mathrm{HCl}$ (10\%) à froid.

Tableau 4: Description morphologique d'un profil type de Cambisols vertiques à Diounkan.

\begin{tabular}{|c|c|c|c|}
\hline Horizon & $\mathbf{A}$ & B & $\mathbf{C}$ \\
\hline Epaisseur (cm) & 0 à 20 & 20 à 160 & 160 à 175 \\
\hline \multirow[t]{2}{*}{ Couleur } & $2,5 \mathrm{Y} 4 / 3$ & $2,5 \mathrm{Y} 4 / 4$ & 2,5 Y 5/6 \\
\hline & brun olive & brun olive & brun olive claire \\
\hline Taches & néant & néant & $\begin{array}{l}\text { environ 5\% de taches } \\
\text { d'oxydo-réduction }\end{array}$ \\
\hline Texture & limono-argileuse & argileuse & argileuse \\
\hline Structure & $\begin{array}{l}\text { polyédrique moyennement } \\
\text { développée et peu } \\
\text { nombreuses faces de } \\
\text { pression }\end{array}$ & $\begin{array}{l}\text { polyédrique grossière très } \\
\text { bien développée à tendance } \\
\text { prismatique et de très } \\
\text { nombreuses faces de } \\
\text { pression }\end{array}$ & $\begin{array}{l}\text { polyédrique à } \\
\text { assemblage compact } \\
\text { et quelques faces de } \\
\text { pression }\end{array}$ \\
\hline Porosité & $\begin{array}{l}\text { nombreux pores tubulaires } \\
\text { et fentes de retrait }\end{array}$ & $\begin{array}{l}\text { nombreux pores tubulaires et } \\
\text { très larges fentes de retrait }\end{array}$ & $\begin{array}{l}\text { très peu de pores } \\
\text { tubulaires }\end{array}$ \\
\hline Refus (\%) & 20 & 7 & 49 \\
\hline $\begin{array}{l}\text { Grav. Ferrug. } \\
(\%)\end{array}$ & 60 & 95 & 50 \\
\hline $\begin{array}{l}\text { Caill. dolom. } \\
(\%)\end{array}$ & 40 & 5 & 50 \\
\hline Consistance & peu dure & dure & très dure \\
\hline Test HCl & pas effervescence & pas effervescence & pas effervescence \\
\hline Transition & progressive & progressive & - \\
\hline
\end{tabular}

Texture (triangle USDA) ; Couleur (Code Munsell) ; Refus : Refus minéral ; Grav. Ferrug.: Graviers ferrugineuses ; Caill. dolom.: Cailloux dolomitiques ; Test HCl: Réaction de la terre fine avec le $\mathrm{HCl}(10 \%)$ à froid. 
Tableau 5: Description morphologique d'un profil type de Cambisols vertiques endogleyiques à Diounkan.

\begin{tabular}{|c|c|c|c|}
\hline Horizon & $\mathbf{A}$ & $\mathbf{B}$ & $\mathbf{C}$ \\
\hline Epaisseur (cm) & 0 à 30 & 30 à 120 & 120 à 140 \\
\hline \multirow[t]{2}{*}{ Couleur } & $10 \mathrm{YR} 3 / 3$ & $2,5 \mathrm{Y} 4 / 4$ & $2,5 \mathrm{Y} 4 / 4$ \\
\hline & brun sombre & brun olive & brun olive \\
\hline Taches & néant & $\begin{array}{l}\text { environ } 30 \% \text { de taches } \\
\text { d'oxydo-réduction }\end{array}$ & $\begin{array}{l}\text { environ } 40 \% \text { de taches } \\
\text { d'oxydo-réduction }\end{array}$ \\
\hline Texture & limono-argilo-sableuse & argileuse & argileuse \\
\hline Structure & $\begin{array}{l}\text { polyédrique sub- } \\
\text { angulaire et peu } \\
\text { nombreuses faces de } \\
\text { pression }\end{array}$ & $\begin{array}{l}\text { polyédrique moyennement } \\
\text { développée et de } \\
\text { nombreuses faces de } \\
\text { pression }\end{array}$ & $\begin{array}{l}\text { polyédrique à } \\
\text { assemblage compact et } \\
\text { quelques faces de } \\
\text { pression }\end{array}$ \\
\hline Porosité & $\begin{array}{l}\text { nombreux pores } \\
\text { tubulaires et fentes de } \\
\text { retrait }\end{array}$ & $\begin{array}{l}\text { nombreux pores tubulaires et } \\
\text { fentes de retrait }\end{array}$ & $\begin{array}{l}\text { quelques pores } \\
\text { tubulaires et fines } \\
\text { fentes de retraits }\end{array}$ \\
\hline Refus (\%) & 3 & 15 & 2 \\
\hline Grav. Ferrug. (\%) & 80 & 100 & 96 \\
\hline Caill. dolom. (\%) & 20 & néant & 4 \\
\hline Consistance & peu dure & très dure & très dure \\
\hline Test HCl & pas effervescence & pas effervescence & pas effervescence \\
\hline Transition & progressive & progressive & - \\
\hline
\end{tabular}

Tableau 6: Description morphologique d'un profil type de Lixisols ferriques sur éboulis latéritiques à Samendéni.

\begin{tabular}{|c|c|c|c|}
\hline Horizon & $\mathbf{A}$ & $\mathbf{B}$ & $\mathbf{C}$ \\
\hline Epaisseur (cm) & 0 à 30 & 30 à 105 & 105 à 150 \\
\hline \multirow[t]{2}{*}{ Couleur } & $5 \mathrm{YR} 4 / 6$ & $5 \mathrm{YR} 5 / 6$ & 7,5 YR 4/6 \\
\hline & rouge jaunâtre & rouge jaunâtre & brun foncé \\
\hline Taches & néant & néant & $\begin{array}{l}\text { environ } 30 \% \text { de taches } \\
\text { d'oxydo-réduction }\end{array}$ \\
\hline Texture & limono-sableuse & argileuse & argileuse \\
\hline Structure & massive & $\begin{array}{l}\text { polyédrique à } \\
\text { assemblage compact }\end{array}$ & $\begin{array}{l}\text { polyédrique à assemblage } \\
\text { compact }\end{array}$ \\
\hline Porosité & $\begin{array}{l}\text { nombreux pores } \\
\text { tubulaires }\end{array}$ & peu de pores tubulaires & très peu de pores tubulaires \\
\hline Refus (\%) & 48 & 37 & 45 \\
\hline Grav. Ferrug. (\%) & 96 & 100 & 95 \\
\hline Caill. dolom. (\%) & 4 & néant & 5 \\
\hline Consistance & peu dure & dure & très dure \\
\hline Test HCl & pas effervescence & pas effervescence & pas effervescence \\
\hline Transition & progressive & discontinue & - \\
\hline
\end{tabular}


Tableau 7: Humidités à la capacité au champ ( $\mathrm{pF} 2,5)$, au point de flétrissement ( $\mathrm{pF} 4,2)$ et la réserve utile (RU) des sols.

\begin{tabular}{|c|c|c|c|c|c|c|}
\hline \multirow[b]{2}{*}{$\begin{array}{c}\text { paramètr } \\
\text { es }\end{array}$} & \multirow[b]{2}{*}{$\begin{array}{c}\text { Horizo } \\
\text { ns }\end{array}$} & \multicolumn{2}{|c|}{$\begin{array}{c}\text { Versants de colline } \\
\text { dolomitique }\end{array}$} & \multicolumn{2}{|c|}{$\begin{array}{c}\text { Plaines et dépressions } \\
\text { périphériques }\end{array}$} & \multirow{2}{*}{\begin{tabular}{|c|}
$\begin{array}{c}\text { Eboulis } \\
\text { latéritiques }\end{array}$ \\
Lixisols \\
ferriques
\end{tabular}} \\
\hline & & $\begin{array}{c}\text { Regosols } \\
\text { colluviques }\end{array}$ & $\begin{array}{c}\text { Cambisols } \\
\text { vertiques } \\
\text { chromiques }\end{array}$ & $\begin{array}{c}\text { Cambisols } \\
\text { vertiques }\end{array}$ & $\begin{array}{c}\text { Cambisols } \\
\text { vertiques } \\
\text { endogleyiques }\end{array}$ & \\
\hline \multirow[t]{3}{*}{ PF2,5 (\%) } & $\mathbf{A}$ & $26,05 \pm 2,81$ & $24,09 \pm 1,41$ & $23,62 \pm 3,06$ & $27,92 \pm 2,79$ & $17,92 \pm 3,81$ \\
\hline & B & $30,03 \pm 1,88$ & $26,68 \pm 2,53$ & $34,67 \pm 2,47$ & $25,73 \pm 3,03$ & $27,74 \pm 2,17$ \\
\hline & $\mathrm{C}$ & $28,14 \pm 1,14$ & $26,77 \pm 3,36$ & $34,18 \pm 4,16$ & $27,23 \pm 5,60$ & $19,67 \pm 3,62$ \\
\hline \multirow[t]{3}{*}{ PF4,2 (\%) } & $\mathbf{A}$ & $20,90 \pm 2,79$ & $19,82 \pm 1,86$ & $19,69 \pm 3,82$ & $24,44 \pm 5,91$ & $15,03 \pm 2,82$ \\
\hline & B & $22,34 \pm 1,68$ & $21,95 \pm 2,43$ & $25,46 \pm 3,30$ & $20,43 \pm 2,15$ & $21,20 \pm 3,69$ \\
\hline & $\mathrm{C}$ & $23,41 \pm 2,21$ & $20,83 \pm 3,53$ & $28,42 \pm 2,87$ & $22,61 \pm 4,58$ & $12,99 \pm 4,96$ \\
\hline \multirow[t]{3}{*}{ RU (mm) } & $\mathbf{A}$ & $18,10 \pm 3,12$ & $17,04 \pm 5,05$ & $17,94 \pm 4,76$ & $15,03 \pm 2,98$ & $14,76 \pm 1,04$ \\
\hline & B & $30,67 \pm 3,92$ & $24,37 \pm 3,65$ & $\begin{array}{c}187,10 \pm \\
6,39\end{array}$ & $86,68 \pm 4,86$ & $89,41 \pm 2,30$ \\
\hline & C & $20,76 \pm 4,64$ & $31,82 \pm 4,84$ & $16,14 \pm 4,32$ & $26,75 \pm 5,73$ & $30,35 \pm 5,08$ \\
\hline
\end{tabular}

Tableau 8: Corrélation entre des paramètres hydriques, granulométriques et chimiques des sols.

\begin{tabular}{|c|c|c|c|c|c|c|c|c|c|c|c|}
\hline & $\mathbf{A}$ & LF & ALF & SG & SF & LG & PF 2,5 & PF 4,2 & RU & MO & CEC \\
\hline A & 1.0000 & & & & & & & & & & \\
\hline $\mathbf{L F}$ & $\begin{array}{c}- \\
0.0928\end{array}$ & 1.0000 & & & & & & & & & \\
\hline ALF & $\begin{array}{c}0.7903 \\
*\end{array}$ & $\begin{array}{c}0.4512 \\
*\end{array}$ & 1.0000 & & & & & & & & \\
\hline SG & $\begin{array}{c}- \\
0.1923\end{array}$ & $\begin{array}{c}- \\
0.2663 \\
*\end{array}$ & $\begin{array}{c}- \\
0.3216 \\
*\end{array}$ & 1.0000 & & & & & & & \\
\hline SF & $\begin{array}{c}- \\
0.6115 \\
*\end{array}$ & $\begin{array}{c}- \\
0.3765 \\
*\end{array}$ & $\begin{array}{c}- \\
0.8203 \\
*\end{array}$ & 0.0897 & 1.0000 & & & & & & \\
\hline LG & $\begin{array}{c}- \\
0.4849 \\
*\end{array}$ & $\begin{array}{c}- \\
0.1069\end{array}$ & $\begin{array}{c}- \\
0.5438 \\
*\end{array}$ & $\begin{array}{c}- \\
0.2554 \\
*\end{array}$ & $\begin{array}{c}0.3929 \\
*\end{array}$ & 1.0000 & & & & & \\
\hline PF 2,5 & $\begin{array}{c}0.4540 \\
*\end{array}$ & $\begin{array}{c}0.3950 \\
*\end{array}$ & $\begin{array}{c}0.6632 \\
*\end{array}$ & $\begin{array}{c}- \\
0.3789 \\
*\end{array}$ & $\begin{array}{c}- \\
0.6585 \\
*\end{array}$ & $\begin{array}{c}- \\
0.2793 \\
*\end{array}$ & 1.0000 & & & & \\
\hline PF 4,2 & $\begin{array}{c}0.3569 \\
*\end{array}$ & $\begin{array}{c}0.4607 \\
*\end{array}$ & $\begin{array}{c}0.5873 \\
*\end{array}$ & $\begin{array}{c}- \\
0.3556 \\
*\end{array}$ & $\begin{array}{c}- \\
0.6063 \\
*\end{array}$ & $\begin{array}{c}- \\
0.1799\end{array}$ & $\begin{array}{c}0.8935 \\
*\end{array}$ & 1.0000 & & & \\
\hline $\mathbf{R U}$ & $\begin{array}{c}0.4360 \\
*\end{array}$ & 0.0171 & $\begin{array}{c}0.4059 \\
*\end{array}$ & $\begin{array}{c}- \\
0.3465 \\
\end{array}$ & $\begin{array}{c}- \\
0.2027\end{array}$ & $\begin{array}{c}- \\
0.3044\end{array}$ & 0.1218 & $\begin{array}{c}- \\
0.0046\end{array}$ & 1.0000 & & \\
\hline
\end{tabular}




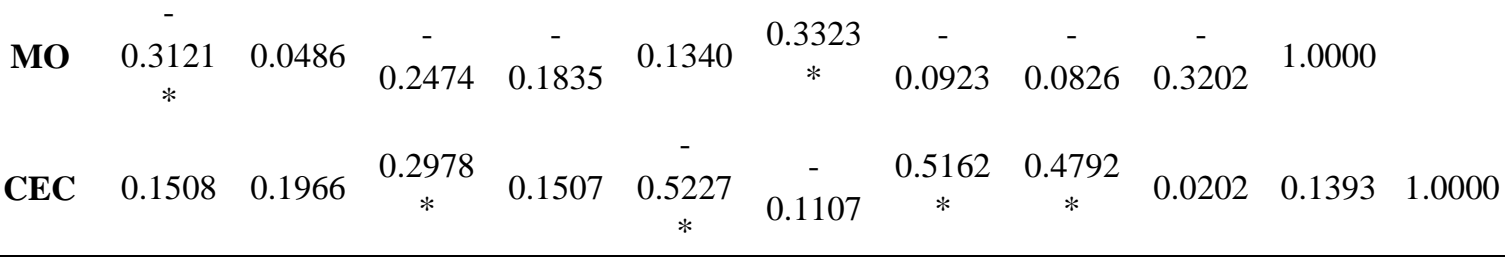

A: argile ; LF: limon fin ; ALF: argile+limon fin ; LG: limon grossier; SF: sable fin ; SG: sable grossier

NB : Les valeurs suivies de * indiquent des corrélations significatives de Spearman au seuil de 5\%.

Tableau 9: Paramètres chimiques des différents types de sols.

\begin{tabular}{|c|c|c|c|c|c|c|}
\hline \multirow[b]{2}{*}{ paramètres } & \multirow[b]{2}{*}{ HZ } & \multicolumn{2}{|c|}{$\begin{array}{l}\text { Versants de colline } \\
\text { dolomitique }\end{array}$} & \multicolumn{2}{|c|}{$\begin{array}{l}\text { Plaines et dépressions } \\
\text { périphériques }\end{array}$} & \multirow{2}{*}{$\begin{array}{l}\text { Eboulis } \\
\text { latéritiques } \\
\text { Lixisols } \\
\text { ferriques }\end{array}$} \\
\hline & & $\begin{array}{l}\text { Regosols } \\
\text { colluviques }\end{array}$ & $\begin{array}{l}\text { Cambisols } \\
\text { vertiques } \\
\text { chromiques }\end{array}$ & $\begin{array}{l}\text { Cambisols } \\
\text { vertiques }\end{array}$ & $\begin{array}{l}\text { Cambisols } \\
\text { vertiques } \\
\text { endogleyiques }\end{array}$ & \\
\hline \multirow[t]{3}{*}{ MO \% } & $\mathbf{A}$ & $1,36 \pm 0,36$ & $1,74 \pm 0,78$ & $1,26 \pm 0,51$ & $0,99 \pm 0,31$ & $1,03 \pm 0,33$ \\
\hline & $\mathbf{B}$ & $0,58 \pm 0,08$ & $0,80 \pm 0,08$ & $0,60 \pm 0,35$ & $0,91 \pm 0,51$ & $0,71 \pm 0,04$ \\
\hline & $\mathbf{C}$ & $0,46 \pm 0,18$ & $0,68 \pm 0,15$ & $0,30 \pm 0,09$ & $0,62 \pm 0,11$ & $0,54 \pm 0,22$ \\
\hline \multirow[t]{3}{*}{$\mathrm{N}$ total $\%$} & $\mathbf{A}$ & $0,07 \pm 0,02$ & $0,08 \pm 0,07$ & $0,07 \pm 0,02$ & $0,06 \pm 0,01$ & $0,06 \pm 0,02$ \\
\hline & $\mathbf{B}$ & $0,03 \pm 0,01$ & $0,05 \pm 0,01$ & $0,04 \pm 0,02$ & $0,05 \pm 0,02$ & $0,04 \pm 0,01$ \\
\hline & $\mathbf{C}$ & $0,02 \pm 0,01$ & $0,04 \pm 0,01$ & $0,02 \pm 0,00$ & $0,03 \pm 0,01$ & $0,03 \pm 0,01$ \\
\hline \multirow[t]{3}{*}{$\mathbf{C} / \mathbf{N}$} & $\mathbf{A}$ & $11,51 \pm 1,10$ & $11,55 \pm 2,44$ & $11,12 \pm 1,24$ & $10,20 \pm 2,34$ & $10,76 \pm 0,96$ \\
\hline & $\mathbf{B}$ & $11,16 \pm 0,52$ & $9,90 \pm 0,55$ & $10,00 \pm 1,12$ & $10,29 \pm 1,61$ & $9,98 \pm 0,61$ \\
\hline & $\mathbf{C}$ & $11,90 \pm 1,27$ & $9,77 \pm 2,17$ & $10,26 \pm 1,41$ & $10,76 \pm 2,00$ & $10,90 \pm 1,15$ \\
\hline \multirow{3}{*}{$\begin{array}{l}\mathrm{BE} \\
\left(\mathrm{cmol}^{(+)} \mathbf{k g}^{-1}\right)\end{array}$} & $\mathbf{A}$ & $11,51 \pm 1,71$ & $8,40 \pm 0,85$ & $6,67 \pm 0,37$ & $7,04 \pm 2,87$ & $4,15 \pm 1,48$ \\
\hline & B & $10,33 \pm 1,19$ & $7,41 \pm 0,72$ & $7,55 \pm 0,00$ & $6,16 \pm 1,74$ & $5,46 \pm 1,87$ \\
\hline & $\mathbf{C}$ & $12,01 \pm 0,47$ & $8,17 \pm 0,44$ & $9,80 \pm 4,26$ & $7,07 \pm 2,04$ & $4,64 \pm 0,33$ \\
\hline \multirow{3}{*}{$\begin{array}{l}\mathrm{Ca}^{2+} \\
\left(\mathrm{cmol}^{(+)} \mathrm{kg}^{-1}\right)\end{array}$} & $\overline{\mathbf{A}}$ & $5,99 \pm 1,83$ & $4,96 \pm 0,91$ & $3,30 \pm 0,94$ & $3,26 \pm 1,25$ & $2,09 \pm 1,07$ \\
\hline & B & $5,01 \pm 0,58$ & $2,28 \pm 0,68$ & $3,27 \pm 0,28$ & $2,40 \pm 0,66$ & $2,22 \pm 0,43$ \\
\hline & $\mathbf{C}$ & $5,84 \pm 0,09$ & $3,34 \pm 1,09$ & $4,04 \pm 1,41$ & $3,07 \pm 0,85$ & $1,88 \pm 0,15$ \\
\hline \multirow{3}{*}{$\begin{array}{l}\mathrm{Mg}^{2+} \\
\left(\mathrm{cmol}^{(+)} \mathrm{kg}^{-1}\right)\end{array}$} & $\mathbf{A}$ & $5,30 \pm 0,43$ & $3,20 \pm 0,98$ & $3,17 \pm 0,99$ & $3,62 \pm 1,58$ & $1,88 \pm 0,86$ \\
\hline & $\mathbf{B}$ & $5,17 \pm 0,61$ & $4,97 \pm 1,06$ & $4,08 \pm 0,18$ & $3,59 \pm 1,14$ & $3,08 \pm 1,39$ \\
\hline & $\mathbf{C}$ & $6,03 \pm 0,41$ & $4,70 \pm 0,66$ & $5,56 \pm 2,81$ & $3,79 \pm 1,18$ & $2,62 \pm 0,50$ \\
\hline \multirow{3}{*}{$\begin{array}{l}\mathrm{K}^{+} \\
\left(\mathrm{cmol}^{(+)} \mathrm{kg}^{-1}\right)\end{array}$} & $\overline{\mathbf{A}}$ & $0,19 \pm 0,04$ & $0,22 \pm 0,06$ & $0,17 \pm 0,02$ & $0,13 \pm 0,03$ & $0,15 \pm 0,04$ \\
\hline & B & $0,10 \pm 0,00$ & $0,12 \pm 0,02$ & $0,16 \pm 0,06$ & $0,12 \pm 0,01$ & $0,13 \pm 0,06$ \\
\hline & $\mathrm{C}$ & $0,11 \pm 0,03$ & $0,10 \pm 0,03$ & $0,15 \pm 0,01$ & $0,15 \pm 0,04$ & $0,11 \pm 0,01$ \\
\hline \multirow{3}{*}{$\begin{array}{l}\mathrm{Na}^{+} \\
\left(\mathrm{cmol}^{(+)} \mathrm{kg}^{-1}\right)\end{array}$} & $\mathbf{A}$ & $0,03 \pm 0,00$ & $0,03 \pm 0,00$ & $0,04 \pm 0,01$ & $0,03 \pm 0,00$ & $0,03 \pm 0,01$ \\
\hline & B & $0,05 \pm 0,01$ & $0,04 \pm 0,02$ & $0,06 \pm 0,01$ & $0,04 \pm 0,01$ & $0,04 \pm 0,01$ \\
\hline & C & $0,04 \pm 0,00$ & $0,03 \pm 0,00$ & $0,06 \pm 0,02$ & $0,07 \pm 0,02$ & $0,03 \pm 0,01$ \\
\hline
\end{tabular}




\begin{tabular}{lllllll}
\hline $\begin{array}{l}\text { CEC } \\
\left(\mathbf{c m o l}^{(+)} \mathbf{k g}^{-1}\right)\end{array}$ & $\mathbf{A}$ & $17,43 \pm 0,87$ & $15,79 \pm 1,46$ & $11,73 \pm 0,96$ & $12,36 \pm 5,87$ & $7,06 \pm 2,03$ \\
\cline { 2 - 7 } & $\mathbf{B}$ & $17,90 \pm 2,13$ & $13,51 \pm 0,76$ & $12,98 \pm 0,28$ & $11,56 \pm 3,89$ & $6,90 \pm 1,97$ \\
\cline { 2 - 7 } & $\mathbf{C}$ & $20,91 \pm 1,77$ & $13,54 \pm 0,77$ & $17,45 \pm 3,39$ & $11,11 \pm 1,60$ & $7,83 \pm 0,67$ \\
\hline $\begin{array}{l}\text { Taux de } \\
\text { saturation } \\
(\%)\end{array}$ & $\mathbf{A}$ & $65,67 \pm 6,51$ & $53,33 \pm 2,08$ & $57,25 \pm 7,02$ & $58,67 \pm 8,14$ & $58,00 \pm 7,51$ \\
\cline { 2 - 7 } & $\mathbf{B}$ & $57,75 \pm 2,35$ & $55,00 \pm 7,57$ & $58,00 \pm 1,41$ & $53,67 \pm 4,51$ & $56,67 \pm 8,10$ \\
\hline pH eau & $\mathbf{C}$ & $57,25 \pm 3,06$ & $60,00 \pm 3,61$ & $57,00 \pm 2,83$ & $62,50 \pm 6,12$ & $59,00 \pm 1,41$ \\
\cline { 2 - 7 } & $\mathbf{A}$ & $6,06 \pm 0,40$ & $5,53 \pm 0,91$ & $5,70 \pm 0,39$ & $5,66 \pm 0,72$ & $6,06 \pm 0,87$ \\
& $\mathbf{C}$ & $5,62 \pm 0,12$ & $5,87 \pm 0,38$ & $5,75 \pm 0,47$ & $5,43 \pm 0,44$ & $5,36 \pm 0,63$ \\
\hline
\end{tabular}

HZ : Horizon ; MO : matière organique ; BE : bases échangeables ; CEC: capacité d'échange cationique

Tableau 10: Ratio des bases échangeables dans les différents types de sol.

\begin{tabular}{|c|c|c|c|c|c|c|}
\hline \multirow{2}{*}{ Rapports } & \multirow[b]{2}{*}{$\begin{array}{l}\text { Horiz } \\
\text { ons }\end{array}$} & \multicolumn{2}{|c|}{$\begin{array}{l}\text { Versants de colline } \\
\text { dolomitique }\end{array}$} & \multicolumn{2}{|c|}{$\begin{array}{l}\text { Plaines et dépressions } \\
\text { périphériques }\end{array}$} & \multirow{2}{*}{$\begin{array}{l}\text { Eboulis } \\
\text { latéritiques } \\
\text { Lixisols } \\
\text { ferriques }\end{array}$} \\
\hline & & $\begin{array}{l}\text { Regosols } \\
\text { colluviques }\end{array}$ & $\begin{array}{l}\text { Cambisols } \\
\text { vertiques } \\
\text { chromiques } \\
\end{array}$ & $\begin{array}{l}\text { Cambisols } \\
\text { vertiques }\end{array}$ & $\begin{array}{l}\text { Cambisols } \\
\text { vertiques } \\
\text { endogleyiques }\end{array}$ & \\
\hline \multirow[t]{3}{*}{$\mathrm{Ca}^{2+} / \mathrm{Mg}^{2+}$} & $\mathbf{A}$ & $1,14 \pm 0,38$ & $1,72 \pm 0,89$ & $1,11 \pm 0,54$ & $0,91 \pm 0,04$ & $1,21 \pm 0,67$ \\
\hline & B & $0,97 \pm 0,00$ & $0,50 \pm 0,27$ & $0,80 \pm 0,10$ & $0,68 \pm 0,09$ & $0,74 \pm 0,35$ \\
\hline & C & $0,97 \pm 0,06$ & $0,71 \pm 0,28$ & $0,76 \pm 0,13$ & $0,82 \pm 0,05$ & $0,72 \pm 0,27$ \\
\hline \multirow[t]{3}{*}{$\mathrm{Mg}^{2+} / \mathrm{K}^{+}$} & $\mathbf{A}$ & $29,81 \pm 5,54$ & $14,70 \pm 1,57$ & $19,63 \pm 4,60$ & $27,03 \pm 6,04$ & $12,98 \pm 2,27$ \\
\hline & B & $50,23 \pm 4,00$ & $43,79 \pm 4,81$ & $28,45 \pm 8,54$ & $31,69 \pm 3,21$ & $24,50 \pm 0,71$ \\
\hline & $\mathbf{C}$ & $55,90 \pm 7,22$ & $49,69 \pm 6,29$ & $37,92 \pm 7,56$ & $25,81 \pm 3,37$ & $23,82 \pm 1,84$ \\
\hline \multirow[t]{3}{*}{$\mathrm{Ca}^{2+} / \mathbf{K}^{+}$} & $\mathbf{A}$ & $31,86 \pm 5,44$ & $25,02 \pm 5,94$ & $19,70 \pm 3,87$ & $24,51 \pm 4,22$ & $13,12 \pm 5,62$ \\
\hline & B & $48,70 \pm 3,82$ & $21,43 \pm 4,92$ & $23,40 \pm 6,39$ & $20,84 \pm 3,96$ & $17,79 \pm 2,56$ \\
\hline & $\mathbf{C}$ & $54,11 \pm 8,82$ & $35,01 \pm 5,86$ & $27,62 \pm 1,36$ & $21,11 \pm 2,99$ & $17,09 \pm 4,34$ \\
\hline \multirow[t]{3}{*}{$\mathrm{K}^{+} / \mathrm{CEC}$} & $\mathbf{A}$ & $0,01 \pm 0,00$ & $0,01 \pm 0,00$ & $0,01 \pm 0,00$ & $0,01 \pm 0,00$ & $0,02 \pm 0,01$ \\
\hline & B & $0,01 \pm 0,00$ & $0,01 \pm 0,00$ & $0,01 \pm 0,00$ & $0,01 \pm 0,00$ & $0,02 \pm 0,01$ \\
\hline & $\mathrm{C}$ & $0,01 \pm 0,00$ & $0,01 \pm 0,00$ & $0,01 \pm 0,00$ & $0,01 \pm 0,00$ & $0,01 \pm 0,00$ \\
\hline
\end{tabular}

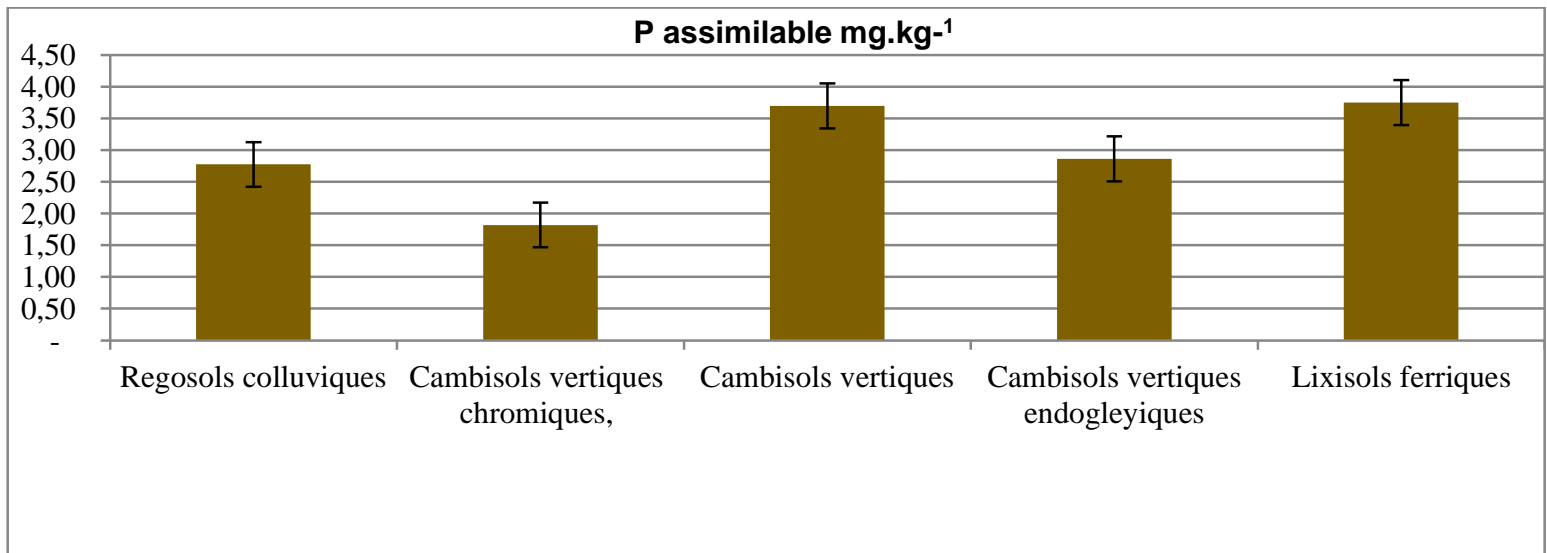

Figure 5: Teneur en phosphore assimilable de l'horizon organo-minéral (A) des sols. 


\section{DISCUSSION}

\section{Caractéristiques physiques des sols}

morphologiques

et

A l'exception des Leptosols lithiques qui n'ont pas été analysés à cause de leur faible représentativité et de leur faible intérêt pour l'agriculture car très superficiels, les sols de versants à pente marquée, peu profonds, sont de couleur rouge du fait du bon drainage favorable à l'oxydation du fer (Kissou et al., 2014). Leurs horizons de profondeur argileux sont parfois compacts ce qui pourrait impacter négativement l'aération du sol, le développement $\mathrm{du}$ système racinaire et l'activité biologique (Garane et al., 2017). En revanche, le nombre élevé de cailloux dolomitiques dans ces sols lourds, pourrait leur fournir par altération de nombreuses bases échangeables nécessaires à leur fertilité chimique. Le test à l'acide $(\mathrm{HCl} 10 \%)$ sur la terre fine est négatif, ce qui suppose l'absence de calcaire actif.

En bas de versant (plaines et dépressions), les taches d'oxydo-réduction sont nombreuses et parfois à environ $30 \mathrm{~cm}$ de la surface (cas des Cambisols vertiques endogleyiques). Ceci dénote une remonté de la nappe en saisons pluvieuses pouvant entrâner des phénomènes d'engorgement nocif à l'aération du sol (Akassimadou et YaoKouamé, 2014). Les argiles gonflantes (type 2/1), soient héritées des dolomies ou synthétisées après la décarbonatation, accumulées dans les sols des plaines et des dépressions périphériques, sont à l'origine des nombreuses fentes de retrait, des faces de pression et de la structure polyédrique. Cette structure parfois bien développée tend à devenir prismatique dans les Cambisols vertiques. (Touhtouh et al., 2014)

En effet, en saison pluvieuse, ces argiles de type $2 / 1$ absorbent une quantité importante d'eau entre leurs feuillets et se gonflent. Ensuite, cette eau s'évapore en saison sèche et les argiles se rétractent en provoquant des fissures dans le sol (Kazmierczak et al., 2016). Les nombreuses fentes de retrait et pores tubulaires créent des conditions idéales d'aération et de développement de la faune du sol.
Les Cambisols qui occupent les niveaux de basses altitudes reçoivent aussi bien des argiles de décarbonatation des dolomies que des graviers ferrugineux d'anciennes cuirasses des plateaux qui les surplombent. Les Lixisols ferriques qui, justement, se sont développés quasi exclusivement sur les éboulis latéritiques, sont caractérisés par une présence massive de cailloux et de graviers ferrugineux provenant de ces anciennes cuirasses. Ces Lixisols ont également une structure massive en surface et polyédrique à assemblage compact en profondeur.

\section{Caractéristiques hydriques des sols}

L'eau utile (RU) des sols étudiés dépend du taux de particules fines (argiles et limons fins) d'une part, car la RU est liée positivement au taux d'éléments fins. D'autre part la hauteur d'eau utile dépend également de l'épaisseur (z) de l'horizon, il est donc normal que les Cambisols vertiques, profonds et riches en éléments fins aient la plus grande RU dans leur horizon B plus épais.

La rétention d'eau dans la microporosité s'explique par l'assemblage des particules fines du sol (Costa et al., 2013). L'étude de Bruand et Tessier (2000) a montré que la teneur en eau aux différents potentiels (PF 2,5 et PF 4,2) dépendait de l'argile, de la MO, mais aussi, de la CEC. Dans les sols étudiés, seules l'argile et la CEC sont liées significativement aux deux humidités caractéristiques. La MO ne varie pas significativement avec ces deux potentiels hydriques. La teneur élevée en argile masquerait l'effet de la MO dans la rétention d'eau surtout lorsque cette dernière est faible (Rawls et al., 2003). Cette remarque est d'autant plus pertinente que le taux de MO faible en surface devient très négligeable en profondeur par rapport à l'argile, d'où la corrélation négative et significative entre ces deux caractéristiques du sol.

\section{Caractéristiques chimiques des sols}

Les carences en matière organique, azote et phosphore assimilable ont été constatées dans tous les sols. Ces déficiences seraient accentuées par le système de mise en 
culture continue sans apport de fertilisants organiques ou organo-minéraux (Gigou et al., 2004; Koulibaly et al., 2016). Les cambisols vertiques endogleyiques situés en bas de versant sont régulièrement sollicités pour les cultures (riz, patate, etc.). Cette utilisation prolongée sans apport de fumure organique expliquerait en partie la baisse excessive de MO dans l'horizon organo-minéral. Au regard du ratio $\mathrm{C} / \mathrm{N}$, la minéralisation de la matière organique est rapide dans l'horizon organominéral. Cependant, les faibles rapports $\mathrm{C} / \mathrm{N}$ dans les horizons (B et C), essentiellement minéraux et pauvres en matière organique, ne traduisent pas forcement une minéralisation rapide de la matière organique dans ces horizons. Ce faible ratio $\mathrm{C} / \mathrm{N}$ en profondeur seraient plutôt liées à un taux d'humification plus important de la matière organique dans les horizons inférieurs des sols. (Grandière et al., 2007).

En dépit des faibles teneurs en MO, tous les sols à l'exception des Lixisols ferriques présentent une CEC élevée (>10 $\left.\mathrm{cmol}^{(+)} \mathrm{kg}^{-1}\right)$ qui indique la présence d'argiles gonflantes de type 2/1 (Kazmierczak et al., 2016). Ces dernières sont, soient hérités (illite), ou issues de la néoformation (smectites) après la décarbonatation. Fabien (2009) indique qu'un taux élevé de $\mathrm{Ca}^{2+} \mathrm{et}_{\mathrm{Mg}^{2+}}$ dans le sol pourrait favoriser la genèse des smectites. Cette faible CEC des Lixisols ferriques rend compte la prédominance des argiles de type 1/1 dont la kaolinite (Ousman et al., 2008).

Les pertes par lixiviation des bases entraînent une désaturation importante du complexe adsorbant dont le corollaire est la tendance à l'acidification des sols. Le taux de saturation élevé dans l'horizon organo-minéral des Regosols s'explique par le nombre élevé de bases $\left(\mathrm{Ca}^{2+}\right.$ et $\left.\mathrm{Mg}^{2+}\right)$. Ces bases proviendraient aussi bien de l'altération des colluvions de dolomie que de l'entrânement par ruissellement, des cations $\mathrm{Ca}^{2+}$ et $\mathrm{Mg}^{2+}$ depuis l'affleurement dolomitique situé en amont.

Les deux cations $\mathrm{Ca}^{2+}$ et $\mathrm{Mg}^{2+}$ libérés par l'altération des dolomies devraient en principe empêcher l'acidification des sols en remplaçant l'ion $\mathrm{H}^{+}$et $\mathrm{Al}^{3+}$ du complexe adsorbant (Koulibaly et al., 2016; Ahukaemere et al., 2018). Cependant, il semble que dans ce milieu semi-humide, les quantités de $\mathrm{Ca}^{2+}$ et $\mathrm{Mg}^{2+}$ libérées sont inférieures aux quantités lixiviées par les eaux de pluie. Toutefois, le $\mathrm{pH}$ légèrement acide ne constitue pas un frein majeur au bon développement de la plupart des plantes (Trigalet et al., 2017).

L'une des contraintes majeures des sols étudiés est la carence en $\mathrm{K}^{+}$sur le complexe adsorbant par rapport à $\mathrm{Ca}^{2+}$ et $\mathrm{Mg}^{2+}$, le magnésium étant particulièrement en excès dans ces sols. Le ratio $\mathrm{Ca}^{2+} / \mathrm{Mg}^{2+}$ est inférieur à 1 à partir de l'horizon médian. Cela s'explique par le taux élevé de magnésium dans la roche mère et la mobilité croissante de $\mathrm{Ca}^{2+}$ par rapport à $\mathrm{Mg}^{2+}$ lors de la décarbonatation et la désaturation des sols (Tableau 1). En effet, le $\mathrm{Mg}^{2+}$ qui entre dans le réseau cristallin des argiles (2/1) est retenu plus longtemps dans le sol que le $\mathrm{Ca}^{2+}$, après la décarbonatation (Fabien, 2009). L'excès de $\mathrm{Mg}^{2+}$ constaté en profondeur pourrait impacter négativement la nutrition des plantes (Hannan, 2011; Akassimadou et Yao-Kouamé, 2014). Ce paramètre est à prendre en compte pour améliorer la rentabilité des sols, car selon la profondeur du sol et de la nappe, les racines de certaines cultures annuelles (maiis, coton, etc.) pratiquées dans la région peuvent parfois aller jusqu'à $100 \mathrm{~cm}$ de profondeur (Chen et al., 2018). Celles des cultures pluriannuelles comme l'anacardier aussi cultivé, sont encore plus profondes.

Une autre conséquence liée à l'excès de magnésium pourrait être la déficience en $\mathrm{K}^{+}$ (Akassimadou et Yao-Kouamé, 2014). En effet, un antagonisme entre magnésium et potassium est susceptible de provoquer une lixiviation de $\mathrm{K}^{+}$(Weis et al., 2009). Dans ces sols, le potassium est en déficit (Koulibaly et al., 2016) par rapport au calcium et au magnésium. Selon (Hannan, 2011), le ratio $\mathrm{Mg}^{2+} / \mathrm{K}^{+}$optimum est compris entre 3 et 4 . L'intervalle optimum pour $\mathrm{Ca}^{2+} / \mathrm{K}^{+}$s'étend de 4 à 17 . La carence en $\mathrm{K}^{+}$pourrait aussi être accentuée par la présence d'argiles gonflantes (2/1) dans les sols (Hamza, 2008). Celles-ci adsorbent facilement l'ion $\mathrm{K}^{+}$dans leur structure interne, le rendant ainsi non disponible. L'influence de $\mathrm{Mg}^{2+}$ est plus 
marquée dans les sols de versants de dolomie (Regosols colluviques et Cambisols vertiques chromiques) du fait de la faible profondeur de la roche dolomitique au niveau de ces versants. L'effet de ce cation et du $\mathrm{Ca}^{2+}$ diminue dans la dépression où la roche dolomitique se trouve plus en profondeur. Les Lixisols rencontrés montrent les plus faibles teneurs en $\mathrm{Mg}^{2+}$ et $\mathrm{Ca}^{2+}$ mais présentent également en profondeur un ratio $\mathrm{Ca}^{2+} / \mathrm{Mg}^{2+}<1$. Ceux-ci seraient influencés par l'apport de magnésium de la dolomie à travers les eaux de ruissellement et les mouvements verticaux de la nappe. Lors de l'exploitation des sols issus des collines dolomitiques, il est fortement recommandé d'apporter régulièrement du potassium sous forme d'engrais potassiques $(\mathrm{KCl})$ ou phosphatés (NPK ou Burkina Phosphate), nécessaires pour relever la teneur en $\mathrm{K}^{+}$, afin de corriger le déséquilibre cationique et la carence en $\mathrm{P}$ assimilable (Lompo et al., 2009; Koulibaly et al., 2016). Les carences en potassium pour les plantes sont souvent liées à un déséquilibre entre les cations (Hannan, 2011 ; Akassimadou et Yao-Kouamé, 2014).

L'apport régulier de la fumure organique reste une solution viable pour améliorer les propriétés des sols régulièrement cultivés pendant de longues années. La fumure organique permettra de réduire le processus d'acidification tout en approvisionnant les sols en nutriments pour accroître le taux de saturation et la CEC (Trigalet et al., 2017).

\section{Conclusion}

L'étude toposéquentielle des caractéristiques morphopédologiques des sols a montré que ceux-ci divergent en fonction de la qualité et/ou de la teneur en argiles issus de roches dolomitiques. Dans les sols peu profonds des versants de collines, les nombreux cailloux dolomitiques en altération constituent un réservoir d'éléments chimiques pour les sols. Les sols en bas de versant deviennent plus foncés et plus profonds avec une structure polyédrique tendant à devenir prismatique. Les nombreuses et larges fentes de retrait contribuent à améliorer la porosité des sols. Les Lixisols ferriques répertoriés se développent sur les "éboulis latéritiques". Les sols de décarbonatation ont des caractéristiques très dépendantes des propriétés chimiques de la dolomie. Les argiles héritées de la dolomie ou celles de néoformation de type $2 / 1$, assurent une meilleure structuration des sols et une CEC moyenne à élevée, malgré la faible teneur en matière organique. L'une des particularités majeures de ces sols issus de la décarbonatation est la prédominance de $\mathrm{Mg}^{2+}$ sur le complexe adsorbant, qui pourrait créer par antagonisme, un déficit significatif en $\mathrm{K}^{+}$. Le potassium et le phosphore assimilable sont deux éléments dont les faibles teneurs pourraient limiter la productivité des sols. Pour une utilisation durable et bénéfique de ces sols, il conviendrait de sélectionner des cultures et des modes de fertilisation adaptées à chaque contexte. Les sols des vallées s'accommodent mieux aux principales cultures de la région notamment le coton, le maïs, le riz pluvial et les arbres fruitiers. Ils pourraient aussi convenir au maraîchage et à la culture de bananier si bien sûr les conditions d'irrigation sont réunies (réalisation de retenues d'eau ou de forages).

\section{CONFLITS D'INTERETS}

Les auteurs déclarent n'avoir aucun conflit d'intérêts.

\section{CONTRIBUTIONS DES AUTEURS}

FK était l'investigateur principal et a participé à toutes les étapes de l'étude et de la rédaction du manuscrit. PNZ a assuré la direction scientifique de ce travail et la correction du manuscrit. EH a contribué à l'organisation des données et à la correction du manuscrit. KO a contribué à la description des sols et à la correction du manuscrit. Il a facilité la détermination des paramètres physiques des sols au laboratoire. TBM a contribué à la description et à l'échantillonnage des sols. Il a aussi apporté des corrections au manuscrit.

\section{REMERCIEMENTS}

Les auteurs remercient les responsables et le personnel des Laboratoires du Bureau des Mines et de la Géologie du Burkina Faso (BUMIGEB); de physique du sol de l'Institut de l'Environnement et de la Recherche Agricole (INERA/Saria) du 
Burkina Faso et du Bureau National des Sols du Burkina Faso (BUNASOLS).

\section{REFERENCES}

Ahmad S, Zaini H, Zaharidah AB. 2009. XRF determination of major elemental contents of clay samples from north-west peninsular Malaysia. Journal of Nuclear and Related Technologies, 6(1): 230-236.

Ahukaemere CM, Aririguzo BN, Onwudike SU. 2018. Elemental ratios and their implications in fertility status of soils of owerri agricultural zone. The International Journal of Agriculture, Management and Technology, 2(1): 4555. http://www.ijamt.com.ng

Akassimadou EF, Yao-Kouamé A. 2014. Caractéristiques morpho-pédologiques et potentiels d'un sol de bas-fond secondaire développé sur granito-gneiss en région de savane guinéenne (Centre de la Côte d'Ivoire). Journal of Applied Biosciences, 79: 6968-6982. DOI: http://dx.doi.org/10.4314/jab.v79i1.17

Baize D, Van Oort F, Nédélec H. 2016. Un facteur majeur de la pédogenèse après décarbonatation dans le cas des formations superficielles calcaires redistribuées au quaternaire: la nature des résidus non carbonatés. Etude et Gestion des Sols, 23(1): 173-192.

Bruand A, Tessier D. 2000. Water retention properties of the clay in soils developed on clayey sediments: Significance of parent material and soil history. European Journal of Soil Science, 51: 679-688.

Bunasols. 2002. Etude morphopédologique de la province du Houet. Echelle 1:100 000, p.53. et annexes.

Chen J, Liu L, Wang Z, Sun H, Zhang Y, Lu Z, Li C. 2018. Determining the effects of nitrogen rate on cotton root growth and distribution with soil cores and minirhizotrons. PLOS ONE, 13(5): 1-14. DOI:

https://doi.org/10.1371/journal.pone.0197284

Costa A, Albuquerque JA, da Costa A, Pértile P, da Silva FR. 2013. Water retention and availability in soils of the state of santa catarina-brazil: effect of textural classes, soil classes and lithology. R. Bras. Ci.

Solo, 37: 1535-1548. DOI : https://doi.org/10.1590/S010006832013000600010

Fabien H. 2009. Modélisation des diffractogrammes de minéraux argileux en assemblages complexes dans deux sols de climat tempéré. Implications minéralogique et pédologique. Thèse de doctorat, Université de Poitiers, France, p. 205.

Garane A, Koussao S, Traoré M, Sawadogo M, Péquénio XP. 2017. Influence de l'itinéraire technique sur les propriétés physiques et hydriques du sol sous culture du blé tendre (Triticum aestivum L.) dans une rotation de «longue durée» dans la zone «non-chernozem» de la Fédération de Russie. Int. J. Biol. Chem. Sci., 11(2): 886-900. http://ajol.info/index.php/ijbcs

Gigou J, Giraudy F, Doucouré COT, Healy S, Traoré KB. 2004. L'âge des champs: un indicateur du passage de la culture itinérante à la culture permanente dans le bassin cotonnier du Mali. Cah. Agric., 13(6): 467-472.

Grandière I, Razafimbelo $T$, Barthès $B$, Blanchart E, Louri J, Ferrer H, Chenu C, Wolf N, Albrecht A, Feller C. 2007. Distribution granulo-densimétrique de la matière organique dans un sol argileux sous semis direct avec couverture végétale des Hautes Terres malgaches. Etude et Gestion des Sols, 14(2): 117-133.

Hamza MA. 2008. Understanding Soil Analysis Data. Resource management technical report 327, Department of Agriculture and Food, Government of Australia. http://www.agric.wa.gov.

Hannan JM. 2011. Potassium-magnesium antagonism in high magnesium vineyard soils. Graduate theses and dissertations. Iowa State University, p.37. https://lib.dr.iastate.edu/etd/12096

Hugot G, Carbonnel JP. 1986. Le Lapiaz de Souroukoudinga (Burkina-Faso): un karst à micro-tourelles d'âge supposé quaternaire. KARSTOLOGIA, 8(2): 3538. http://www.persee.fr/doc/karst_07517688_1986_num_8_1_2138. 
IUSS Working Group WRB. 2015. World Reference Base for Soil Resources 2014, Update 2015. International Soil Classification System for Naming Soils and Creating Legends for Soil Maps. World Soil Resources Reports 106, Rome, p.192. http://www.fao.org/3/i3794en/I3794en.p df

Jonquet P. 1963. Prospection des dolomies dans le sédimentaire ouest-voltaïque. BRGM., secteur Haute-Volta, 55p.multi.

Kazmierczak J-B, Maison T, Laouafa F, Delalain P, Fleureau J-M. 2016. Un nouveau dispositif pour la caractérisation du retrait et du gonflement des sols argileux. Rev. Fr. Geotech., 147(1). DOI: https://doi.org/10.1051/geotech/2016005

Kissou R, Traore E, Gnakambary Z, Nacro H B, Sedogo MP. 2014. Connaissance endogène de la classification et de la fertilité des sols en zone SudSoudanienne du Burkina Faso. VertigO, 14 (1). DOI: 10.4000/vertigo. 14616

Koulibaly B, Traore O, Dakuo D, Lalsaga R, Lompo F, Zombré P N. 2014. Acidification des sols ferrugineux et ferrallitiques dans les systèmes de production cotonnière au Burkina Faso. Int. J. Biol. Chem. Sci., 8(6): 2879-2890. http://ajol.info/index.php/ijbcs

Koulibaly B, Dakuo D, Traore M, Traore O, Nacro HB, Lompo F, Sédogo MP. 2016. Effets de la fertilisation potassique des sols ferrugineux tropicaux sur la nutrition minérale et la productivité du cotonnier (Gossypium hirsutum L.) au Burkina Faso. Int. J. Biol. Chem. Sci., 10(2):722736. http://ajol.info/index.php/ijbcs

Lompo F, Segda Z, Gnankambary Z, Ouandaogo N. 2009. Influence des phosphates naturels sur la qualité et la biodégradation d'un compost de pailles de maïs. Tropicultura, 27(2): 105-109. https://www.tropicultura.org
Ousman Z, Alassane A, Dan LN, Rumori P, Gemma TP, Issaka A. 2008. Caractérisation des Sols de Périmètres Irrigués de l'Ouest du Niger par Diffraction de Rayons X. J. Soc; OuestAfr. Chim ; 026: 89 - 97. https://www.soachim.org

Prudente D, Baize D, Dubois J-P. 2002. Le cadmium naturel dans une forêt du haut Jura français. In Les Éléments Traces Métalliques dans les Sols - Approches Fonctionnelles et Spatiales, Baize D et Tercé M (eds). INRA Éditions: Paris; 5370.

Rawls WJ, Pachepsky YA, Ritchie JC, Sobecki TM, Bloodworth H. 2003. Effect of Soil Organic Carbon on Soil Water Retention. Geoderma, 116: 61-76. DOI: https://doi.org/10.1016/S0016706(03)00094-6

Touhtouh D, Moujahid Y, El Faleh EM, EL Halimi R. 2014. Caractérisations Physico-chimiques de trois types de sols du Sais, Maroc. J. Mater. Environ. Sci., 5(5): 1524-1534. http//www.jmaterenvironsci.com

Trigalet S, Chartin C, Krüger I, Carnol M, Oost KV, Wesemael BV. 2017. Soil organic carbon fractionation for improving agricultural soil quality assessment a case study in Southern Belgium (Wallonia). Biotechnol. Agron. Soc. Environ, 21(3): 191-200.

Walkley A, Black IA. 1934. An examination of the Degtjareff method for determining soil organic matter, and a proposed modification of the chromic acid titration method. Soil Science, 37(1): 29-38.

Weis W, Gruber A, Huber C, Göttlein A. 2009. Element concentrations and storage in the aboveground biomass of limed and unlimed Norway spruce trees at Höglwald. European Journal of Forest Research, 128: 437-445. DOI: 10.1007/s10342-009-0291-5. 